\title{
Enhancing Short-Circuit Level and Dynamic Reactive Power Exchange in GB Transmission Networks under Low Inertia Scenarios
}

\author{
Dimitrios Tzelepis \\ EEE Department \\ University of Strathclyde \\ Glasgow, UK \\ dimitrios.tzelepis@strath.ac.uk
}

\author{
Qiteng Hong \\ EEE Department \\ University of Strathclyde \\ Glasgow, UK \\ q.hong@strath.ac.uk
}

\author{
Jayaraman Ramachandran \\ NationalgridESO \\ National Grid \\ Warwick, UK
}

jay.ramachandran@nationalgrideso.com

\author{
Campbell Booth \\ EEE Department \\ University of Strathclyde \\ Glasgow, UK
}

\author{
Panagiotis N. Papadopoulos \\ EEE Department \\ University of Strathclyde \\ Glasgow, UK
}

\author{
Guangya Yang \\ Dept. of Electrical Engineering \\ Technical University of Denmark \\ Kgs. Lyngby, Denmark \\ gyy@elektro.dtu.dk
}

\begin{abstract}
The objective of the studies presented in this paper is to demonstrate that the deployment and operational control of Synchronous Condensers (SynCons) combined with Static Compensators (STATCOM) in the GB transmission system can mitigate a part of the challenges associated with the high penetration of renewable energy sources. The case studies include scenarios such as transmission-level faults, fault level calculation and dynamic reactive power provision. For these scenarios, SynCons and STATCOMs of different capacity and design are installed at different regions of the GB transmission system. For these studies, verified models of SynCons and STATCOMs are deployed which are integrated to a representative GB network model. All the studies have been implemented in RMS simulation environment using Power Factory - DIgSILENT package software.
\end{abstract}

Index Terms-Synchronous Condensers, Static Compensators, Transmission Line Faults

\section{INTRODUCTION}

Traditional power systems are gradually evolving towards decarbonised modern power networks. From the power generation perspective, centralised conventional synchronous generation plants are gradually replaced by renewable generation, connected both to transmission and distribution level. In Great Britain (GB), the generation capacity could increase from $103 \mathrm{GW}$ (today) to $189 \mathrm{GW}-268 \mathrm{GW}$ by 2050 , with the more renewable scenarios requiring the highest capacities (with $65 \%$ of generation being local) [1].

As such, the future generation mix in the wider GB system (and specifically in Scotland) will mainly consist of intermittent, asynchronous renewable energy sources. A major challenge of most renewable energy sources (e.g. domestic

This research is supported by SP Energy Networks and NationalGridESO Grid under the PHOENIX project (reference: SPTEN03/Phoenix). solar installations) is that they are largely uncontrollable by system operators under the present operating schemes and other commercial services.

This change in generation mix creates significant system issues which may lead to certain stability risks on the system that need to be managed and also risk the security of supply to GB customers. These issues include the following:

1) Reduced system inertia: a) compromises the network stability and security in the event of a large power imbalance (i.e. loss of load and/or generation), b) results in large frequency deviations and consequently high Rate of Change of Frequency (RoCoF) which can initiate further loss of generation due to the disconnection of distributed generation.

2) Lower Short Circuit Level (SCL): a) increases the risk of commutation failure in Line Commutated Converterbased (LCC) High Voltage Direct Current (HVDC) interconnectors, b) potential risks of compromising protection performance and c) results in poor power quality in conjunction with low performance of phaselocked loop-driven generation.

3) Limited voltage control: in absence of immediate dynamic response (conventionally obtained from large synchronous generators) can result in voltages and voltage angles outside the desired limits.

In order to address these new challenges of lower system inertia and SCL, technological solutions, such as Synchronous Condensers (SynCons) are being encountered. These technologies can replace the rotating machines of the decommissioned generation plants (or synchronous generators can be converted into SynCons). SynCons have been used widely in power systems accounting for reactive support and 
voltage stability [2], [3]. SynCons have also the ability to elevate the SCL which can improve system interconnections (both HVAC and HVDC) [4] and power system protection [5]. Furthermore, SynCons have been found to be remarkably useful in load shedding schemes [6] and primary frequency control and stability [7], [8]. SynCon installations can be realised either by conventional generators [9], [10] or superconducting units [11]

Apart from SynCons, different technologies such as Flexible AC Transmission Systems (FACTS) are being promoted to account for quick control, active harmonic damping, limitation of flicker phenomena and medium to high frequency oscillation damping [12]. As such, these compensating devices could be one of the options to mitigate challenges associated with high penetration of renewable energy sources (but not necessarily the only options). Specifically, the combination of various compensation technologies have the potential to enable subsequent applications to:

- Boost system inertia.

- Provide dynamic voltage regulation.

- Reactive power injection support to alleviate voltage dip conditions.

- Reactive power absorption to mitigate potential overvoltage scenarios in light load conditions.

- Increase the system SCL and system total strength.

- Enhance the oscillation damping capability.

- Aid in maintaining power quality of the network.

This paper investigates and quantifies the impact of SynCons and Static Compensators (STATCOMs) to SCL contribution accounting for short-circuit power and peak shortcircuit current. Their impact on voltage is also assessed by their dynamic reactive power exchange.

\section{System Modelling}

\section{A. GB Transmission System}

The studies presented in this paper are based on a model of national electricity transmission system of GB which is represented by a 36-bus equivalent network [13]. The model has been developed by NationalgridESO in DIgSILENT PowerFactory and the network topology is depicted in Fig. 1.

Each numbered node in the model represents a part of the system and consists of a mix of different energy sources and loads. Generators within each zone are represented by static generators and synchronous machines including relevant dynamic controllers. In each zone, generation, loads, HVDC interconnectors and transmission lines are connected to 400$\mathrm{kV}$ busbars.

\section{B. SynCon \& STATCOM Units}

For the studies presented in this paper SynCon and STATCOM units have been modelled in DIgSILENT PowerFactory and then integrated to the 36-bus GB network model (refer to Fig. 1). Both units are connected to a $400-\mathrm{kV}$ busbar as illustrated in Fig. 2

The SynCon is modelled as a standard synchronous machine without a governor. Three control systems have been integrated

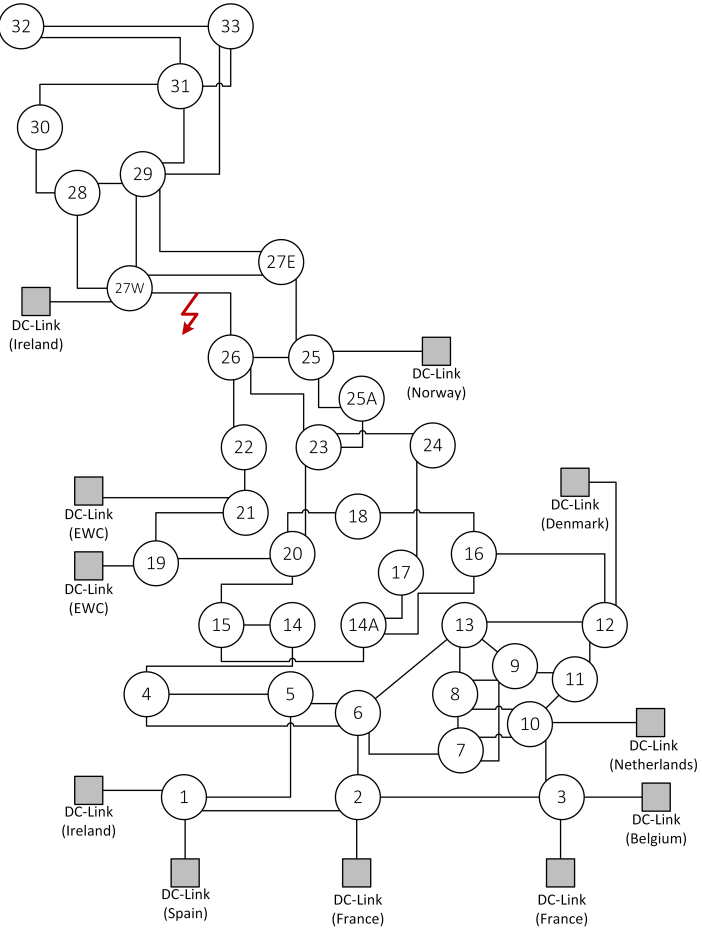

Fig. 1. Transmission network of GB: 36-zone equivalent.

which include i) Automatic Voltage Regulator (AVR), ii) Power System Stabiliser (PSS) and iii) Over Excitation Limiter (OEL).

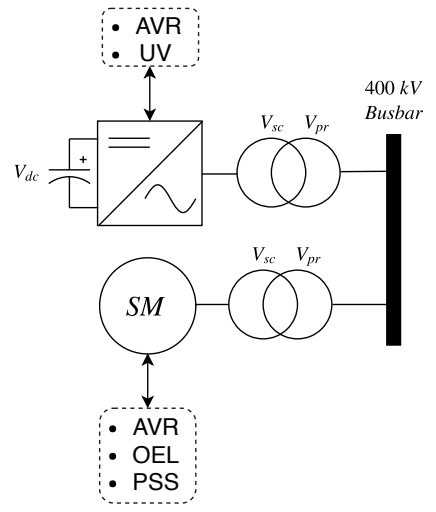

Fig. 2. Schematic of SynCom and STATCOM units connected to a $400 \mathrm{kV}$ busbar.

The core part of the STATCOM model is the VSC source, which represents the STATCOM as a controllable current source. The voltage control is based on a closed-loop system with control of the positive-sequence voltage at the STATCOM high voltage bus. The voltage regulator is required to be fast enough to counteract voltage variations and disturbances, but also retain an adequate stability margin. An under-voltage (UV) strategy is deployed in the model to achieve a voltage ride through capability during UV conditions and also to avoid transient over-voltages when the system voltage recovers 
from a voltage drop. To support the harmonics analysis, the STATCOM together with its filter has been modelled as a Norton equivalent under harmonic frequencies. This is realised by implementing the harmonic data in the static generator model which represents the VSC source.

\section{SCL Calculation}

A major part of this paper is associated with the contribution of SynCon and STATCOMs to the SCL. As such, it is of utmost important to understand and define the method for calculating of the SCL. The studies reported in this paper utilise the superposition method for the SCL calculation. In this method, the pre-fault load conditions of the network are considered. Therefore, the load flow and the setting of the transformer tap changers are a pre-requisite.
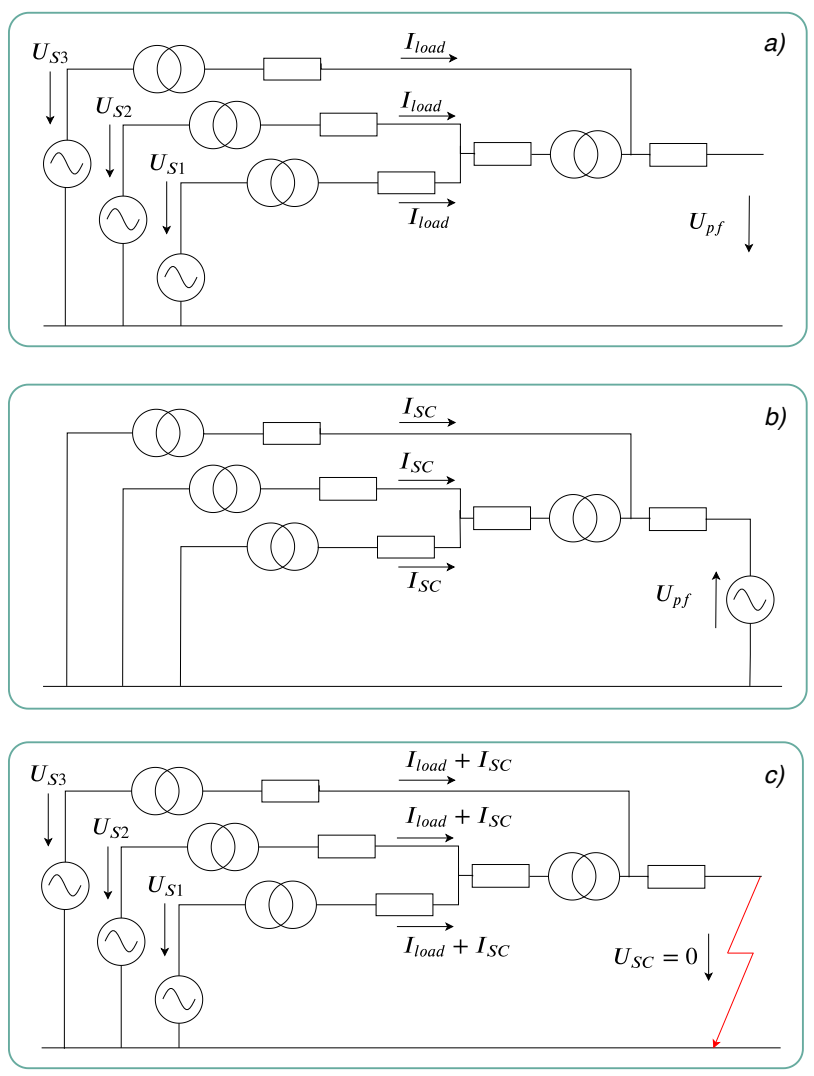

Fig. 3. Stages of SCL calculation using super-position method: a) pre-fault condition, b) pure-fault condition, c) post-fault condition.

As depicted in Fig. 3 the entire method analyses the fault behaviour into three discrete steps:

- Pre-fault: The procedure begins with the calculation of the normal operating conditions (refer to Fig. 3a). These conditions reflect the excitation of generators, tap changer position of transformers, load conditions and breakers status. In this step the pre-fault voltage $U_{p f}$ at the fault location is determined.

- Pure-fault: In the second step (refer to Fig. 3b) a negative version of pre-fault voltage $U_{p f}$ is applied to the fault location and all other sources are set to zero. Practically, $U_{p f}$ is the only voltage source in the network.

- Post-fault: Finally, the system performance after fault inception is determined by overlaying (complex adding) both the pre-fault and pure-fault conditions (refer to Fig. 3c).

\section{Simulation Results}

The simulation results presented in this section are extracted from both static and dynamic simulations. In particular, Section III-A presents the static SCL analysis while Section III-B presents the dynamic reactive power exchange studies.

\section{A. Short Circuit studies}

In order to calculate the contribution of SynCon units to the SCL, a set of simulation scenarios has been setup to incrementally change the capacity of SynCon units at each zone. The maximum capacity has been set to 700 MVA with increments of $70 \mathrm{MVA}$. The short-circuit power $S_{k}$ and shortcircuit peak current $I_{p}$ have been captured. It should be noted that the control system of STATCOM behaves in such a way to limit the output current to 1.0 p.u. In that sense, the contribution from STATCOM units to the SCL is practically zero and thus no studies considering STATCOM and SCL have been conducted.

The results for SynCon units can be depicted in Fig. 4 and Fig. 5 for $S_{k}$ and $I_{p}$ respectively.

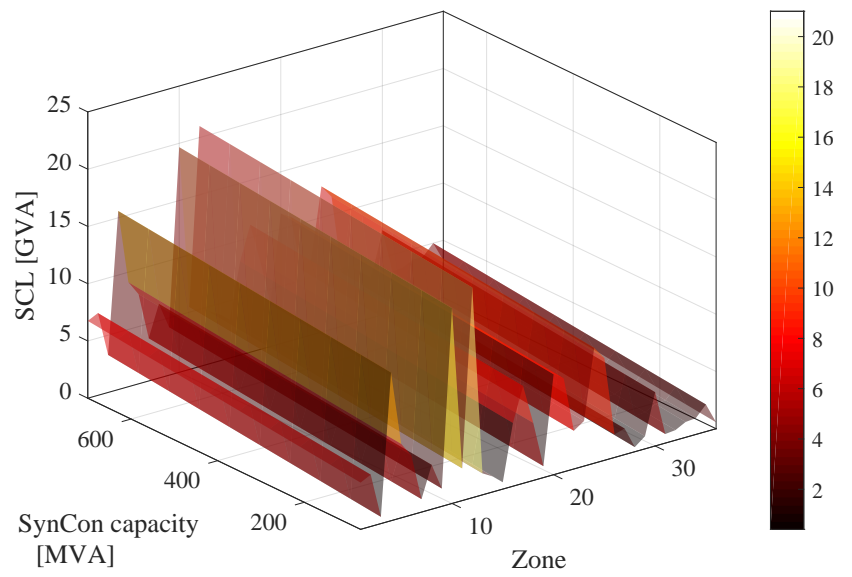

Fig. 4. SCL results accounting for short-circuit power $S_{k}$ at different zones and for different SynCon capacity.

From Fig. 4 and Fig. 5 it can be seen that the addition of SynCon units can definitely increase both short-circuit power $S_{k}$ and short-circuit peak current $I_{p}$. The greatest SCL relevant boost has been observed in Zone 26, where a 700 MVA SynCon elevated the $S_{k}$ from 406.4 MVA to 3102.5 MVA and $I_{p}$ from $1.5 \mathrm{kA}$ to $11.5 \mathrm{kA}$. It shall be highlighted that the SCL is of utmost importance as the declining of SCL in low-inertia systems can is directly associated with challenges related to transmission protection and instability of units based on phaselocked loops [14]. Such a decline can be mainly observed to 


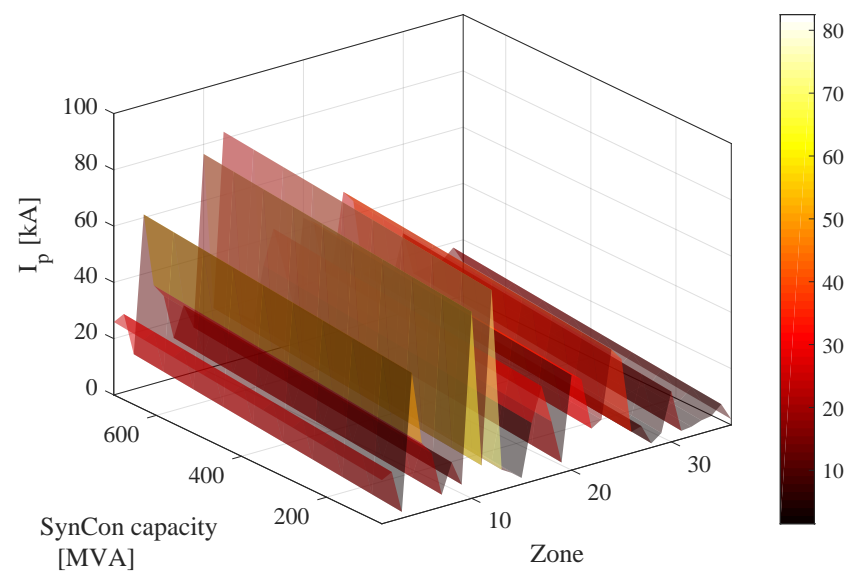

Fig. 5. SCL results accounting for short-circuit peak current $I_{p}$ at different zones and for different SynCon capacity.

Zone 26 to Zone 33 which corresponds to north part of UK including Scotland.

\section{B. Dynamic Reactive Power Support}

In order to assess the reactive power support from SynCon and STATCOM units, studies have been carried out in RMS simulation environment. A three-phase solid fault has been triggered on the line connecting Zone 26 and Zone $27 \mathrm{~W}$ (refer to Fig. 1) which has been cleared after $110 \mathrm{~ms}$. SynCon and STATCOM units have been considered at Zone 25 and Zone 26 under the following case studies:

- Case 1: No support

- Case 2: SynCon (700 MVA)

- Case 3: STATCOM (700 MVA)

- Case 4: SynCon (350 MVA) + STATCOM (350 MVA)

The graphical representation of the system response for Case 2 to Case 4 is depicted in Fig. 6 to Fig. 8 respectively. For easiness of presentation voltage profiles are only presented for Zone 26 which is also the zone closer to the fault. Voltage profile at Zone 26 is also used as a base-case for voltage comparison when SynCom and STACOM units are enabled (i.e. Case to Case 4).

By observing the voltage profile of Zone 26 (refer to Fig. 6(a), Fig. 7(a) and Fig. 8(a)) it can be seen that by utilising SynCon and STATCOM units, the voltage can be slightly improved. Such an improvement can be observed in three different regions accounting for residual-voltage (i.e. fault duration), transient over-voltage (i.e. instant of fault clearance) and over-voltage (i.e. post-fault).

The corresponding reactive power support from SynCom units can be seen in Fig. 6(b) and Fig. 6(c) for Case 2 and in Fig. 8(b) and Fig. 8(c) for Case 4. Reactive power injection can be observed during the fault but also after the fault clearance. Due to the fact that Zone 26 is closer to the fault, the reactive power emmanating from SynCon at Zone 26 is more pronounced.
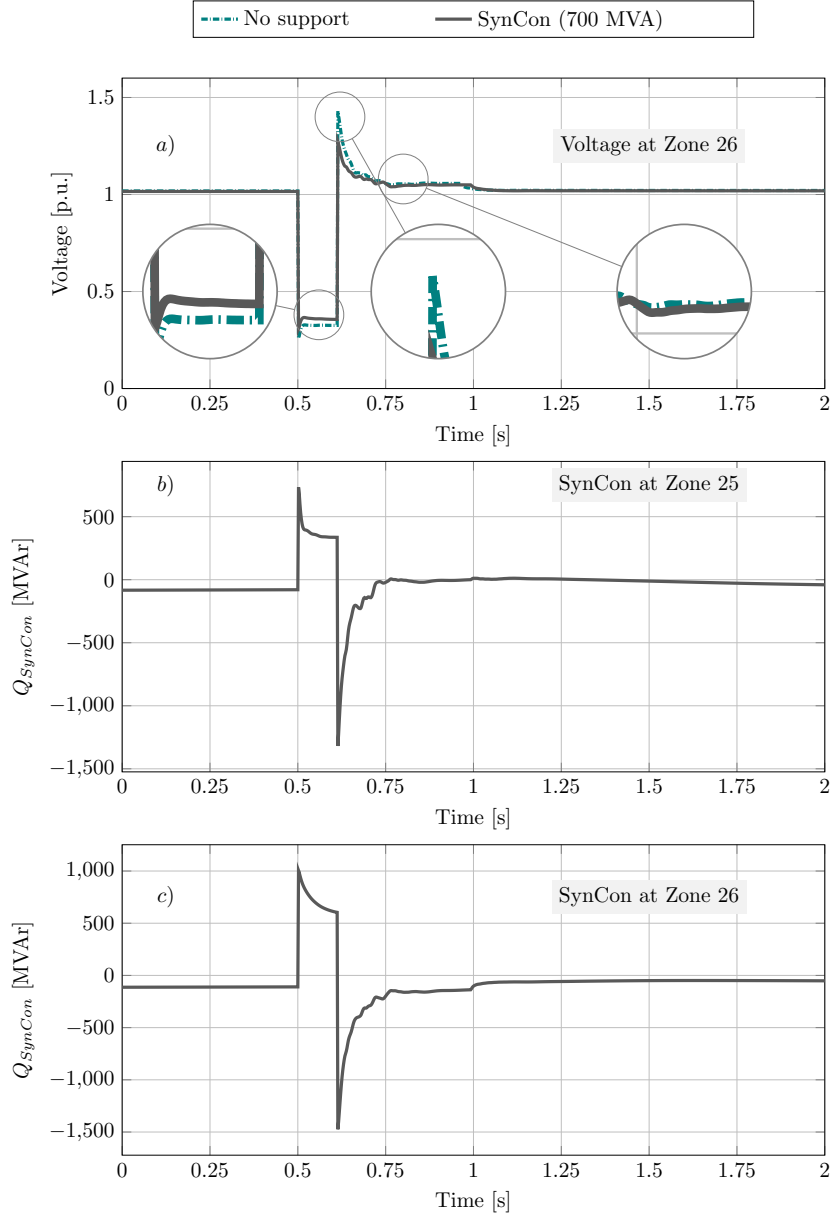

Fig. 6. Voltage profiles at Zone 26 and reactive power support from SynCon units (Case 2).

The corresponding reactive power support from STATCOM units can be seen in Fig. 7(b) and Fig. 7(c) for Case 3 and in Fig. 8(d) and Fig. 8(e) for Case 4. Similarly to the SynCon units, reactive power injection from STATCOM units located at Zone 26 is greater.

It is worth to point out that the instantaneous reactive power injection from SynCon at fault inception is significantly larger than that delivered by the STATCOM. This is due to the fact the design nature of SynCon units allows them to be overloaded for a finite period of time. On the contrary, the reactive power from STATCOM is limited as the output reactive current is held to maximum 1.0 p.u.

Additionally, it has been observed that there is an overshoot of the instantaneous reactive power from STACOM at the instant of fault clearance. This emanates from the fact that STATCOM response is dictated by its control system. Specifically, the under/over-voltage strategy of STATCOM has a time delay which forces the STATCOM to hold the reactive current at 1.0 p.u. Considering that there is transient overvoltage at the instant of fault clearance, the total exported reactive power during the transient over-voltage period is more pronounced. 

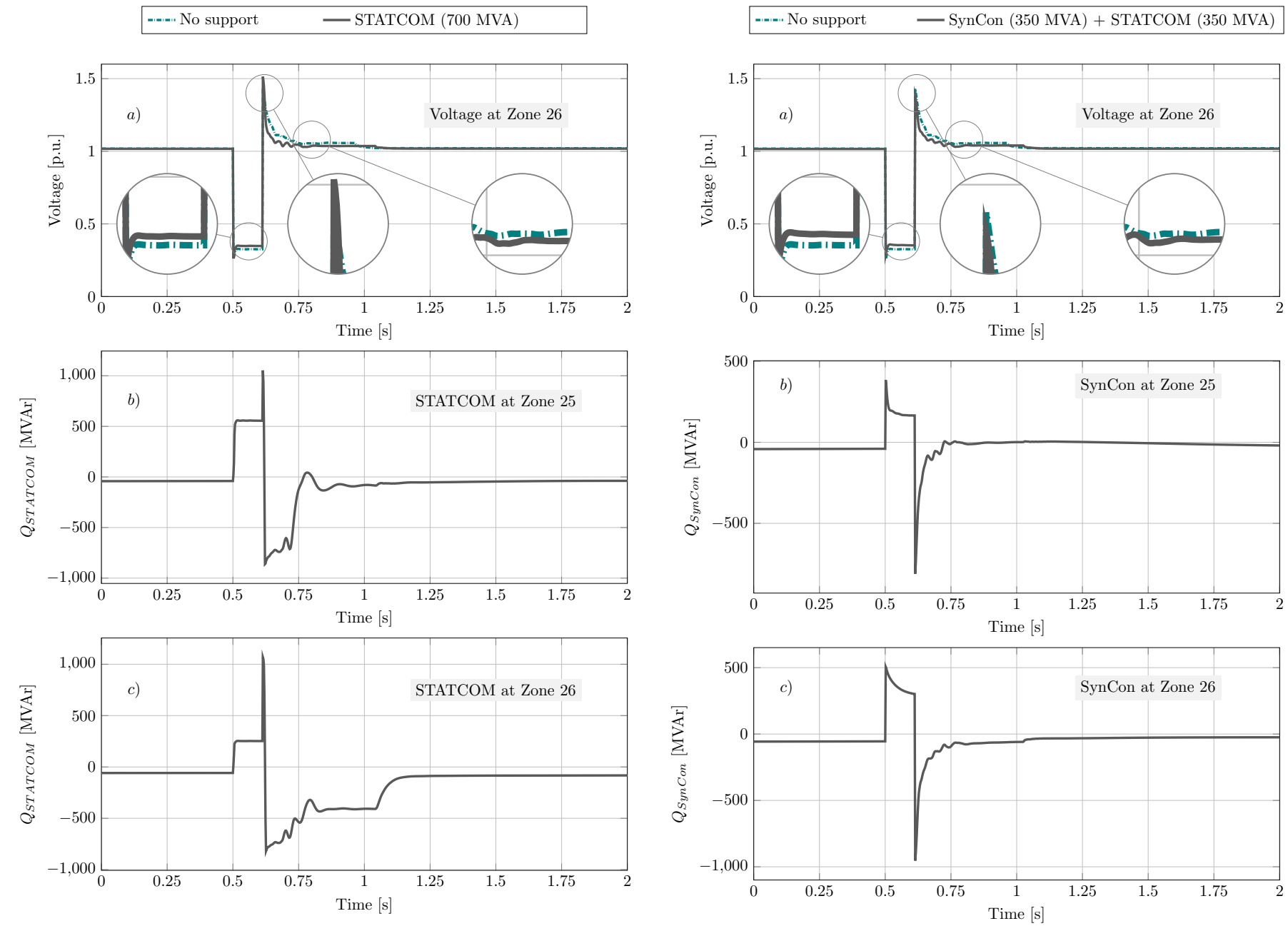

Fig. 7. Voltage profiles at Zone 26 and reactive power support from STATCOM units (Case 3).

\section{COnClusions}

In this paper Synchronous Condenser (SynCon) and Static Compensators (STATCOM) units have been considered to mitigate challenges associated with the high penetration of renewable energy sources.

The deployment and operational control of SynCon and STATOM units in the GB transmission system has been found to be beneficial for the system. In particular, the utilisation of SynCon and STATOM units have been found to increase the short circuit level (in the case of SynCon only) and provide reactive power support during and after three-phase transmission line faults.

It is anticipated that SynCon and STATOM units can further contribute to challenges arising from reduced system inertia (e.g. large frequency deviations in the event of a large loadgeneration imbalance), reduced system short circuit level (e.g. risk of commutation failures in line commutated converter) and enhanced power transfer capability.
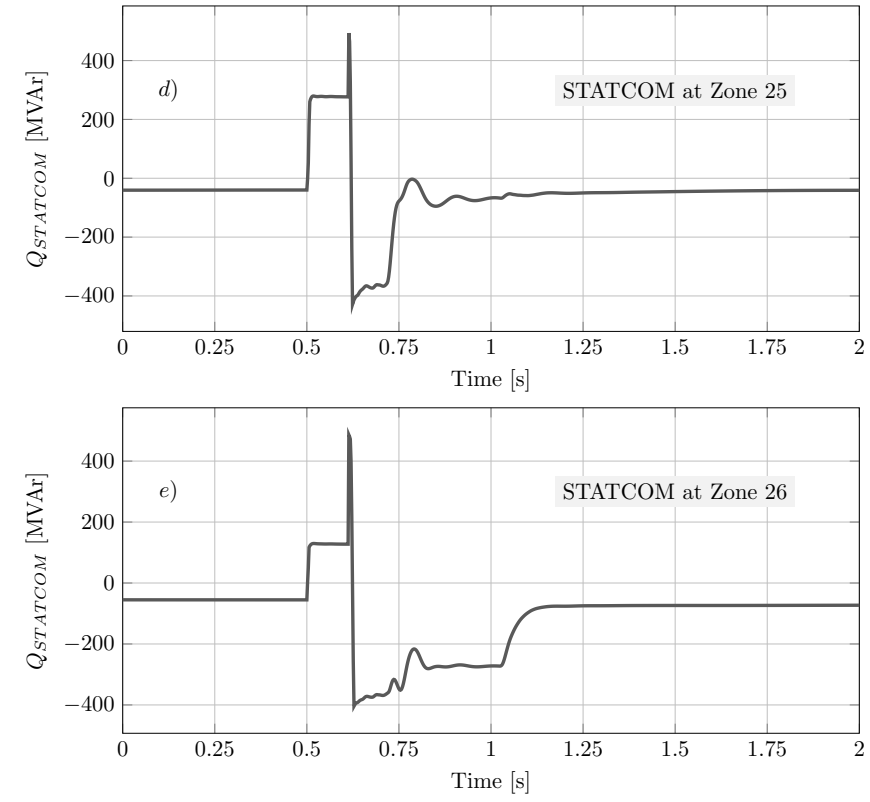

Fig. 8. Voltage profiles at Zone 26 and reactive power support from SynCon and STATCOM units (Case 4). 


\section{REFERENCES}

[1] "Future energy scenarios," National Grid ESO, Tech. Rep., July 2018.

[2] N. Mendis, K. M. Muttaqi, and S. Perera, "Management of batterysupercapacitor hybrid energy storage and synchronous condenser for isolated operation of PMSG based variable-speed wind turbine generating systems," IEEE Transactions on Smart Grid, vol. 5, no. 2, pp. 944-953, March 2014.

[3] F. O. Igbinovia, G. Fandi, I. Ahmad, Z. Muller, and J. Tlusty, "Modeling and simulation of the anticipated effects of the synchronous condenser on an electric-power network with participating wind plants," Sustainability, vol. 10, no. 12, 2018.

[4] E. Ghahremani, A. Heniche, M. Perron, M. Racine, S. Landry, and H. Akremi, "A detailed presentation of an innovative local and widearea special protection scheme to avoid voltage collapse: From proof of concept to grid implementation," IEEE Transactions on Smart Grid, pp. $1-1,2018$.

[5] J. Jia, G. Yang, A. H. Nielsen, and P. Rønne-Hansen, "Impact of VSC control strategies and incorporation of synchronous condensers on distance protection under unbalanced faults," IEEE Transactions on Industrial Electronics, vol. 66, no. 2, pp. 1108-1118, Feb 2019.

[6] R. Yan, T. K. Saha, and N. Modi, "Frequency response and its enhancement using synchronous condensers in presence of high wind penetration," in EEE Power Energy Society General Meeting, July 2015, pp. $1-5$.

[7] A. Moeini and I. Kamwa, "Analytical concepts for reactive power based primary frequency control in power systems," IEEE Transactions on Power Systems, vol. 31, no. 6, pp. 4217-4230, Nov 2016.

[8] H. T. Nguyen, G. Yang, A. H. Nielsen, and P. H. Jensen, "Combination of synchronous condenser and synthetic inertia for frequency stability enhancement in low inertia systems," IEEE Transactions on Sustainable Energy, vol. 'Early Access', pp. 1-1, 2018.

[9] P. E. Marken, M. Henderson, D. LaForest, J. Skliutas, J. Roedel, and T. Campbell, "Selection of synchronous condenser technology for the granite substation," in IEEE PES T D 2010, April 2010, pp. 1-6.

[10] J. Skliutas, D. LaForest, R. D'Aquila, D. Derr, and E. Kronbeck, "Nextgeneration synchronous condenser installation at the VELCO granite substation," in 2009 IEEE Power Energy Society General Meeting, July 2009, pp. 1-8.

[11] S. S. Kalsi, D. Madura, G. Snitchler, M. Ross, J. Voccio, and M. Ingram, "Discussion of test results of a superconductor synchronous condenser on a utility grid," IEEE Transactions on Applied Superconductivity, vol. 17, no. 2, pp. 2026-2029, June 2007.

[12] B. Singh, R. Saha, A. Chandra, and K. Al-Haddad, "Static synchronous compensators (statcom): a review," IET Power Electronics, vol. 2, no. 4, pp. 297-324, July 2009.

[13] M. Yu, A. Dyśko, A. Roscoe, C. Booth, R. Ierna, H. Urdal, and J. Zhu, "Effects of swing equation-based inertial response (sebir) control on penetration limits of non-synchronous generation in the gb power system," in International Conference on Renewable Power Generation, Oct 2015, pp. 1-6.

[14] "System operability network - impact of declining short circuit currents," National Grid ESO, Tech. Rep., December 2018. 